\title{
Summary of transformer partial discharge detection methods
}

\author{
Li Yanda \\ North China Electric Power University, Baoding 071003, China \\ yanda325221@163.com
}

Keywords: Space charge discharging; Transformer oil; Detection method;Partial discharge

\begin{abstract}
In this article, I summarized three main methods about measuring space charge discharging through the physical and chemical effects. It introduced the transformer oil discharging space charge measurement method and its distribution characteristics, and mainly presented gas chromatography detection method, the electric pulse detection method and UHF detection method, and their measurement circuit. Meanwhile I showed the measured waveform diagram.Then I analyzed the advantages and disadvantages of each method and application. Finally, I gave the partial distribution characteristics of the discharging space charge in transformer.
\end{abstract}

\section{Introduction}

Transformer oil referred to oil, so this article refers the transformer oil as an object, and we make a general description of the transformer. Oil discharge space charge is generated in the long run due to the transformer insulating oil wet process and the water is heated to generate gas , then bubbles are formed. When the field strength reaches a certain value, it will space charge discharges and partial discharges. Oil will be accompanied by a series of space charge and discharge of the physical and chemical changes, such as electricity, light, sound, heat and so on. Measurement methods resulting discharge are mainly the following two categories, a non-electrical measuring method, an electrical measuring method. Non-electrical measurement categories include ultrasonic method, light effect measurement, and gas chromatography method; Electrical Measurement categories include electrical pulse detection method, UHF detection. Nowadays, China is more commonly used pulse current method and ultrasonic detection. This article will be described in detail for the gas chromatography method, the electric pulse detection and UHF detection.

\section{Non-electrical measuring methods}

\section{The gas chromatography method}

Oil space charge and discharge,and it will break down, resulting in a lot of gas, you can determine the appropriate type of partial fault detection based on the composition and content of the produced gas, which is gas chromatography method. The main gas after oil cracking is $H_{2}$ and $\mathrm{CH}_{4}$. Gas chromatography has a lot of advantages, such as against electrical interference, cost-effective performance and high sensitivity. So it has been more widely used in line inspection of the transformer. But oil and gas analysis also has many disadvantages: As there will be a long-term through oil and gas analysis testing process, although it is more sensitive to detect latent faults, it can not reflect the sudden fault, and it can be only for qualitative analysis, let alone judge quantificationally.

\section{Electrical measuring methods}

\section{The electric pulse detection}

Pulse current method is a method that detecting transformer bushing non screen grounding wire, chassis ground wire, core ground wire and winding partial discharge causing pulse current is detected by the impedance, consequently getting some basic amount of partial discharge (for instance depending on the discharge volume, the number of discharges and discharges phase). This 
method is the study of the earliest and most widely used method for detecting. IEC developed specifically for this standard. The method has high sensitivity, quantitative measurement of the partial discharge characteristic parameters. It also can determine the partial discharge position of the acoustic signals through the electro-acoustic positioning method.

Pulse current method, is the voltage at both ends of the test product to be mutated into a pulse current in detection circuit. According pulse current, we can calculate the apparent amount of discharge. The so-called apparent discharge value, refers to partial discharge, the test voltage variation across $\Delta V$. If a certain amount of charge is injected at both ends of the test sample $C_{x}$, so that the test sample with a partial discharge generates the same amount of change, this injected charge amount, meaning partial discharge depending on the discharge amount $q$, then $q=C_{x} \Delta V$. Depending discharge amount characterizes the partial discharge intensity.

Here are the pulse current method of testing the circuit shown in Figure 1.

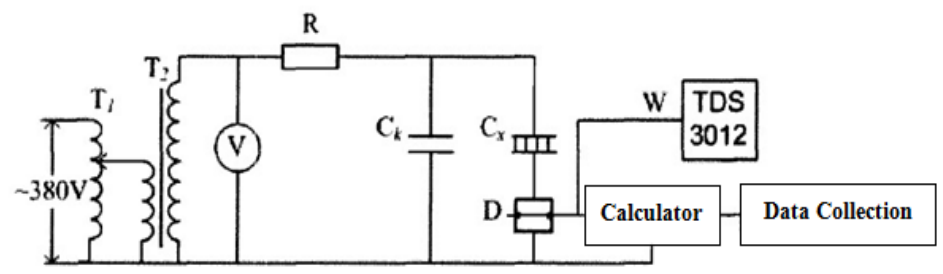

\section{Fig.1 Discharge test circuit}

$T_{1}$ is self-coupling voltage regulator, $T_{2}$ is regulating transformer $100 \mathrm{~V} / 110 \mathrm{KV}, C_{k}$ is coupling capacitor, $C_{x}$ is the test, W:is Tektronix TDS3012 oscilloscope, which role is high frequency limiting and blocking, meanwhile $C_{k}$ has played a role in a pulse current to provide a low impedance path. Voltmeter $\mathrm{V}$ is responsible for measuring experiments provided voltage value, controlling experiment voltage. $\mathrm{D}$ is a current sensor, which has high sensitivity and linearity.

Through the results of the noise reduction and image processing,we can get the following two figures. Fig. 2 is a complex waveform diagram because of the complexity of the field caused by electromagnetic interference.

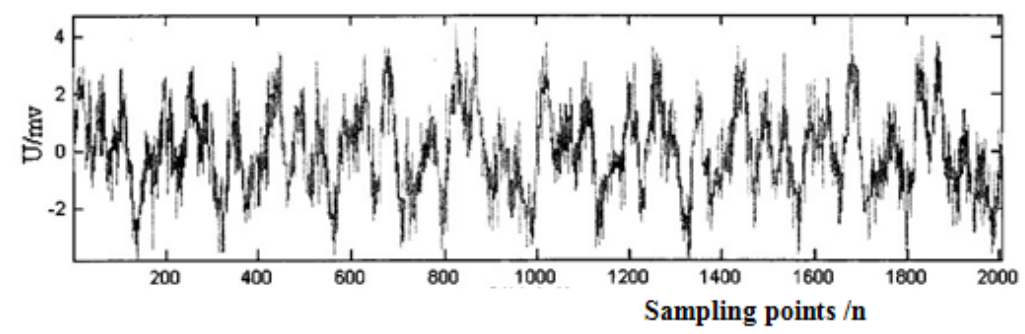

Fig.2 Not after discharge signal processing

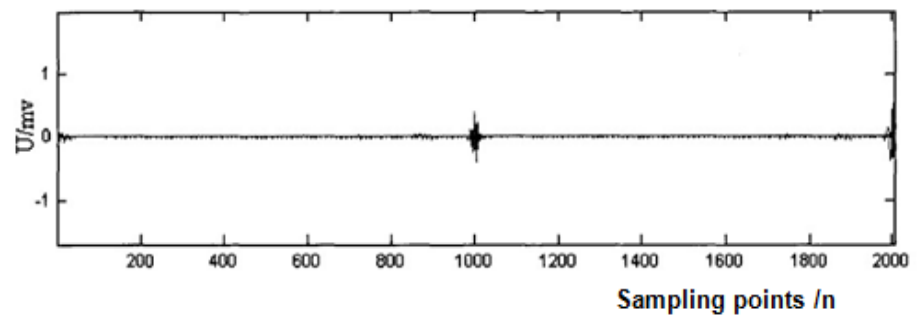

Fig.3 Space charge and discharge signal was obtained after treatment 


\section{UHF detection}

UHF partial discharge detection is a new detection method emerged in recent years, aiming at traditional detection methods disadvantages,. UHF method forms by detecting the internal transformer partial discharge generated by UHF (300 $3000 \mathrm{MHz})$ electromagnetic signal, turning out partial discharge detection and location to achieve immunity.

Detection principle of this method: Each partial discharge occurred in the positive and negative charge, accompanied by a sharp current pulse, and radiated electromagnetic waves around. The spectral characteristics of the partial discharge and electromagnetic wave radiated relative to partial discharge source geometry and the dielectric strength of the discharge gap.Transformer oil has a clapboard structure generally radiate electromagnetic waves up to several GHz. The frequency interference is not higher than the general scene $400 \mathrm{MHz}$, so the UHF detection method can effectively avoid the kinds of electromagnetic interference.

Now as the detection system and the results, I do a general description. Figure 4 is the system detected after experimental power output from the auto regulator T1 through isolation transformer $\mathrm{T} 2$ and received no partial discharge tests on high voltage transformer T3. Isolation transformer T2 may effectively suppress higher harmonic grid fleeing improve the quality of power supply. Figure 5 is an electromagnetic signal detected by the UHF spectrum sensor to the discharge point excitation spectrum analyzer records.

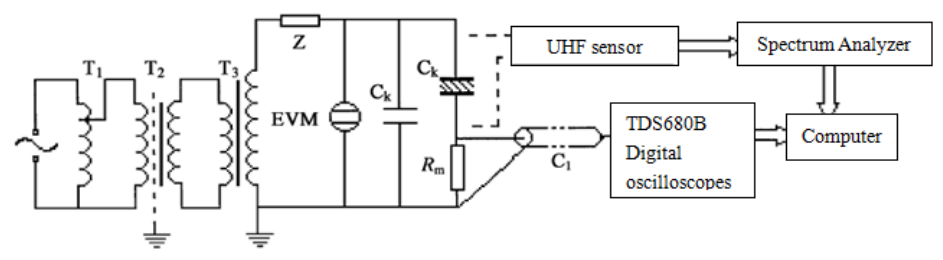

Fig.4 UHF partial discharge detection system

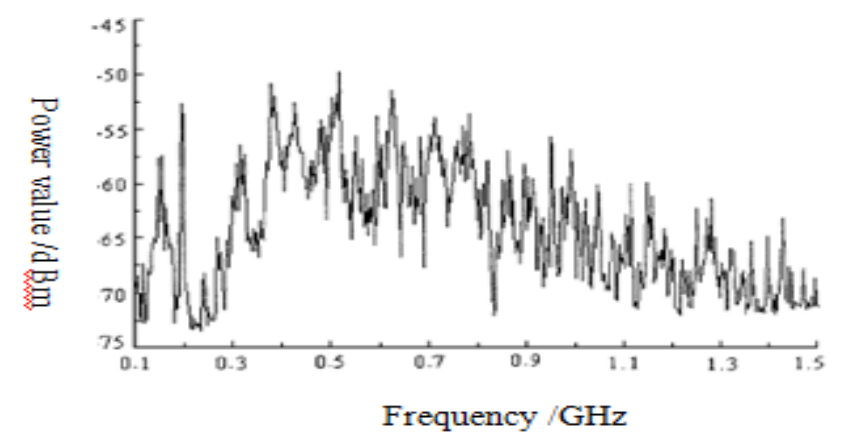

Fig.5 UHF sensor detects the electromagnetic wave was excited discharge

\section{Conclusion}

This paper summarizes three detection methods that measures transformer partial discharging, including that the electric pulse method has used the most widely.Light effect measurement is also largely remain in the laboratory stage, and gas chromatography is a relatively early measurement method which has some certain applications . UHF detection method, for its strong anti-jamming capability, has a measure promising. 


\section{References}

[1] Wu Peng, Experimental study of typical power transformer discharge model, high-voltage electrical, 2004, 40 (3): 161-163..

[2] Li Yanqing, Ultrasonic Detection power transformer partial discharge, Beijing: North Electric Power University, 2003.

[3] Xu Yang, Partial discharge measurement and optical pulse and electrical measurement method, high-voltage technology, 27 (4): 3-5.

[4] Zhao Xiaohui, Oil partial discharge detection pulse current method compared with UHF method, high-voltage technology .2008,34 (7): 1401-1404.

[5] Chen Qingguo, Transformer oil UHF partial discharge detection test study, high-voltage technology, 2002,28 (12): 23-25. 